\title{
Look Twice before You Clamp: Decapitation of an Omphaloenteric Duct
}

\section{A Case Report}

\author{
Cornelia van Tuil Amulya K. Saxena Günter H. Willital \\ Pediatric Surgical University Clinic, Münster, Germany
}

\section{Key Words}

Persistent omphaloenteric duct - Congenital umbilical cord hernia - Trauma to intestinal loops - Clamping of umbilical cord $\cdot$ Delivery

\begin{abstract}
Objective: To report a case of accidental decapitation of a persistent omphaloenteric duct (POD) in congenital hernia of the umbilical cord in a newborn male. Case Presentation and Intervention: The newborn was transferred to an intensive care unit, where under closer inspection the diagnosis of an omphalocele was presumed. Surgical exploration at our Pediatric Surgical University Clinic revealed a clamped and decapitated POD in the umbilical cord. The decapitation had gone unnoticed due to severe asphyxia resulting from meconium aspiration encountered at the time of delivery. Using general anesthesia, the clamp was removed under sterile conditions. The decapitation of the POD in the herniated umbilical cord was confirmed at this time. The severed intestine was sutured and closed using 5-0 Vicryl sutures. The further course was uneventful. Conclusion: Herniation of the umbilical cord at the time of birth by intestinal loops or POD is rare, but caution should be taken by midwives before clamping in order to avoid the type of injury seen in this case.
\end{abstract}

Copyright (C) 2006 S. Karger AG, Basel

\section{KARGER}

Fax +4161306 1234

E-Mail karger@karger.ch

www.karger.com
C 2006 S. Karger AG, Basel

1011-7571/06/0152-0156\$23.50/0

Accessible online at:

www.karger.com/mpp

\section{Introduction}

Anterior abdominal wall defects at the time of birth can present either as gastroschisis or omphalocele. Gastroschisis presents with herniation of the fetal abdominal contents into the amniotic fluid without any covering. Omphalocele, on the other hand, is a congenital anterior abdominal wall defect at the base of an umbilical cord with herniation of the abdominal contents covered by the parietal peritoneum, the amnion and Wharton's jelly. However, mild forms of omphalocele can present as congenital hernia of the umbilical cord (CHUC) with herniation of either the solitary intestinal loop or persistent omphaloenteric duct (POD). In embryological terms the omphaloenteric duct is a patent intestinal loop in the fetal umbilical cord which regresses and is obliterated at the time of birth or persists as a residual Meckel's diverticulum. The risk factors in omphalocele as well as mild forms such as CHUC are still not completely known and their association with other birth defects has not always been established. The significance of POD in CHUC in newborns has been noted in the literature [1]. Traumatic injury to intestinal loops in CHUC has also been reported and emphasized in order to prevent this type of injury at the time of birth. We report such a case of accidental umbilical clamp decapitation of a POD in the herniated umbilical cord. 
Fig. 1. Schematic representation of decapitated POD in a CHUC (a) and histological presentation of the decapitated POD (b).

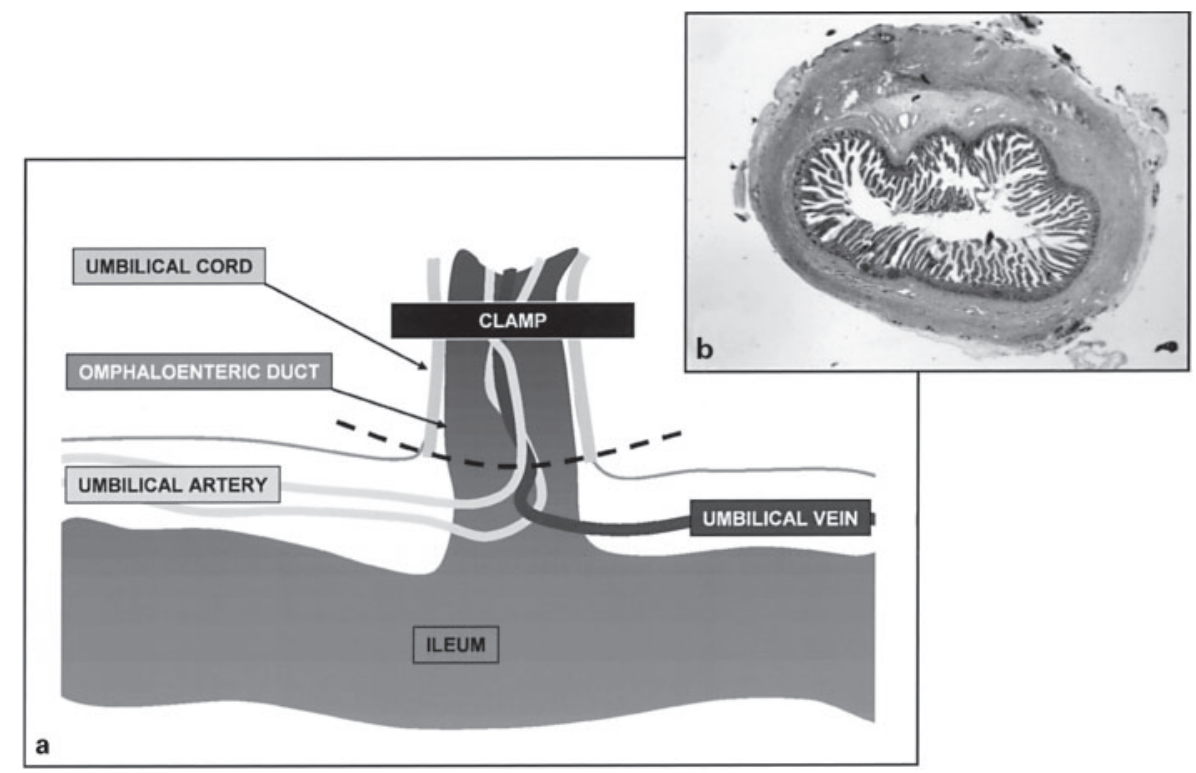

\section{Case Report}

A newborn male delivered in the 40th week of gestation presented with asphyxia, presumed to be due to meconium aspiration. The newborn was transferred to an intensive care unit for further observation. Clinical examinations revealed an unusual thickening of the umbilical cord. The cord was clamped approximately $7 \mathrm{~cm}$ from the umbilical base by the midwife after delivery. The residual umbilical cord was approximately $2 \mathrm{~cm}$ in diameter. This clinical finding led to the presumption of a small but previously undiagnosed omphalocele. Ultrasound examinations during the entire course of pregnancy and 2 weeks before delivery, however, had not demonstrated any sign of abnormality in the area of the umbilicus. On careful examination, a trapped severed intestinal loop could be vaguely identified in the CHUC. The newborn was then transferred in a stable condition to the Pediatric Surgical University Clinic and an immediate decision was taken for the operative exploration of the umbilical cord. During the surgical procedure, a POD was found adherent to the umbilical cord. The top of the POD was decapitated and crushed by the umbilical clamp. The adherent POD was dissected free from Wharton's jelly and resected at its base on the intestinal loop (fig. 1). At the point of resection, the intestinal loop was sutured transversely using 5-0 Vicryl interrupted sutures. The further course was uneventful as there was no further complication. The specimen was sent for pathological evaluation, and the presence of a severed POD was clearly evident (fig. 1).

\section{Discussion}

Careful inspection of the umbilical cord of every newborn at the time of delivery is essential. Any unusual thickening of the base of the cord or other anomalies should alert the obstetricians, pediatricians, and nurses, who customarily clamp, manipulate or shorten the cord as a routine procedure at the time of delivery. The existence of anomalies or associated structures within the umbilical cord has frequently been reported in the literature $[2,3]$. Injury to such structures that may be trapped in the inconspicuous umbilical cord may occur during routine clamping if such herniations are not searched for.

Prenatal ultrasound examinations play a critical role in the diagnosis of fetal anomalies and have influenced the management of newborns at the time of delivery. Accurate prenatal diagnosis of anterior abdominal wall defects is generally possible during routine prenatal ultrasound examinations and these anomalies are rarely misdiagnosed. Awareness of characteristic ultrasound manifestations facilitates therapy and prognosis $[4,5]$. Despite advances made in prenatal ultrasound diagnostics, CHUC, which is the minimal form of umbilical cord herniation, can go unnoticed. This was evident in the case presented, where a CHUC went unnoticed until the 38th week of gestation.

Even when ultrasound examinations do not detect a CHUC, the presence of a single loop of intestine or POD may be quite challenging at the time of delivery $[6,7]$. Delivery of uncomplicated pregnancies by midwifes is common, and newborns with CHUC pose a high-risk group for herniated structure injuries. These injuries may also go primarily unnoticed. It is only later, after careful evaluation, that their presence may be discovered. If an 
unusual umbilical cord thickening is suspected, the patient should be carefully evaluated by a pediatric surgeon with experience in neonatal surgery. Further medical treatment or surgical intervention should be left to his discretion. However, timely surgical exploration and intervention in suspected cases help to avoid complications and reduce morbidity.

If a POD in a CHUC is confirmed, careful dissection and separation of the POD from Wharton's jelly should be undertaken. Once the POD is freed, it must be traced to its base on the intestinal loop, which in most cases is the proximal ileum. We prefer to resect the POD longitudinally at its base and suture the intestinal opening using interrupted transverse sutures. The POD may contain ectopic tissues similar to those found in Meckel's diverticulum and partial resection is unadvisable. The entire procedure can be carried out without any skin incisions. Once the intestinal loop has been sutured and returned into the abdominal cavity, an umbilical plastic recon- struction using pursestring Vicryl suture is sufficient to achieve the end result.

This mode of surgical approach enables quick resolution of an emergency situation along with excellent esthetic results. No skin incisions are necessary, which further markedly reduces the need for postoperative analgesics.

To our knowledge, traumatic decapitation of a POD in the umbilical cord and its successful management have not been reported in the literature.

\section{Conclusion}

The danger of traumatic injury to incarcerated structures may at first go unnoticed in rare cases of CHUC. In suspected cases, it is recommended to perform higher clamping, as well as follow-up with further investigations and surgical intervention if required.

\section{References}

1 Jona JZ: Congenital hernia of the cord and associated patent omphalomesenteric duct: a frequent neonatal problem? Am J Perinatol 1996; 13:223-226.

$\checkmark 2$ Donlon CR, Furdon SA, Clark DA: Look before you clamp: delivery room examination of the umbilical cord. Adv Neonatal Care 2002; 2:19-26. $\checkmark 3$ Donlon CR, Furdon SA: Assessment of the umbilical cord outside of the delivery room. Part 2. Adv Neonatal Care 2002;2:187-197.

$\checkmark 4$ Fogata ML, Collins HB 2nd, Wagner CW, Angtuaco TL: Prenatal diagnosis of complicated abdominal wall defects. Curr Probl Diagn Radiol 1999;28:101-128.

$\checkmark 5$ Hata T, Aoki S, Hata K, Miyazaki K: Threedimensional ultrasonographic assessment of the umbilical cord during the 2 nd and 3 rd trimesters of pregnancy. Gynecol Obstet Invest 1998;45:159-164.
-6 Bukhonkin MD, Egamberdiev BE: Strangulation of a portion of the ileum with a ligature in a newborn infant with an embryonic umbilical cord hernia. Vestn Khir Im I I Grek 1979;123: 86.

7 Tejani A, Dobias B, Varma P: Intestinal laceration secondary to clamping of an occult omphalocele. Obstet Gynecol 1978;52:483-485. 